\title{
Multiplet-changing Auger transitions in valence double photoionization
}

\author{
U. Becker, O. Hemmers, B. Langer, I. Lee, ${ }^{*}$ A. Menzel, and R. Wehlitz \\ Fritz-Haber-Institut der Max-Planck-Gesellschaft, W-1000 Berlin 33, Germany \\ M. Ya. Amusia \\ Institut für Theoretische Physik, Universität Frankfurt, W-6000 Frankfurt 11, Germany
}

(Received 27 October 1992)

\begin{abstract}
The decay of valence satellite states in neon above the first double-ionization threshold has been studied experimentally and theoretically. Special emphasis was given to differentiate between two decay modes: valence Auger and valence-multiplet Auger decay. It is shown that the latter process is predominant in the low kinetic energy part of the spectrum. The main structures of this low-energy Auger spectrum could be designated by help of calculated transition energies and decay rates.
\end{abstract}

PACS number(s): 32.80.Fb, 32.80.Hd, 32.80.Dz, 33.60.Cv

Double ionization of valence shells in atoms and molecules has recently attracted considerable interest [1-3]. The reason for this recovered, still increasing interest is due to the existence of a delicate balance of competing processes contributing to the valence-shell double ionization. These processes are known as simultaneous and sequential or direct and indirect processes. Whereas the first experiments on double ionization assumed almost all intensity of doubly charged ions resulting from singlephoton ionization as due to a simultaneous or direct double-ionization process, the so-called shakeoff process, the more recent studies showed that this is not true: sequential or indirect processes contribute significantly to the valence double-ionization rate [4-6]. Since that time, experimental and theoretical studies have tried to explore this phenomenon in more and more detail. Armen and Larkins [7] were the first to our knowledge who showed on the basis of neon data from Becker et al. [4] that the sequential processes split into two distinct decay modes: inner-valence and valence multiplet-changing Auger transitions. In both cases the excited electron does participate in the transition process, corresponding to decays where the excited core changes either its electronic configurations [for instance, $\mathrm{Ne}^{+} 2 s 2 p^{5}\left({ }^{3} P\right) n p\left({ }^{2} S\right)$ $\left.\rightarrow \mathrm{Ne}^{2+} 2 s^{2} 2 p^{4}\left({ }^{1} S+{ }^{1} D\right)+e^{-}\right]$or its multiplet coupling [such as $\mathrm{Ne}^{+} 2 s^{2} 2 p^{4}\left({ }^{1} S\right) n p\left({ }^{2} \mathrm{P}\right) \rightarrow \mathrm{Ne}^{2+} 2 s^{2} 2 p^{4}\left({ }^{3} \mathrm{P}\right)$ $\left.+e^{-}\right]$, for example, in a spin-flip process, using hypothetical one-electron levels for the sake of simplicity. Figure 1 shows the different processes schematically for the case of neon. The theoretical and experimental interest is focused on the latter process for two reasons: (i) its high transition probability, and (ii) the occurrence of electrons with very low kinetic energy.

The valence multiplet-changing transition, also referred to as nonresonant autoionization, may, in the extreme, give rise to the ejection of $0-V$ kinetic energy Auger electrons. Figure 2 shows a complete electron spectrum of neon taken at a photon energy of $h v=120$ $\mathrm{eV}$ exhibiting clear evidence for all three processes: simultaneous double ionization, inner-valence Auger transitions, and valence multiplet-changing Auger transi- tions, the latter depicted by the hatched area. The deconvolution of the different processes will be explained in detail elsewhere; here we will concentrate on the appearance and interpretation of the valence multiplet-changing Auger transitions [8]. For this purpose we present highresolution valence multiplet-changing Auger spectra of neon and interpret them by means of designations obtained from calculated energy positions and decay rates. We show that most of the observed Auger transitions may be clearly identified in this way and can be related to the decay of satellite transitions recorded recently with similar resolution on the photoelectron side behind an undulator-wiggler beam line.

The experiments were performed at the Hamburger Synchrotronstrahlungslabor HASYLAB using photons from the 5.6-m toroidal grating monochromator of beamline D4. As in all our former experiments on this subject,

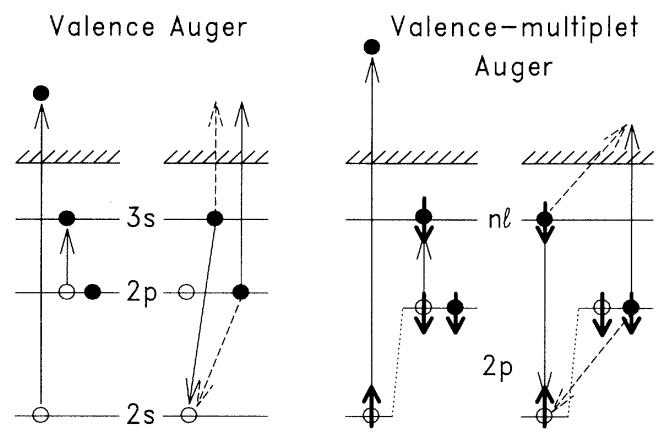

FIG. 1. Simplified schematic representation of neon innervalence $2 s$ photoionization with accompanying excitation together with possible subsequent recombination processes. The inner-valence process utilizes energy obtained from filling the $2 s$ hole to eject an Auger electron $\epsilon l$ (valence Auger). In the case of neon outer-valence $2 p$ photoionization, the valence-multiplet process utilizes the multiplet splitting of the valence hole configuration to provide the Auger energy $\epsilon$ necessary to eject another valence electron, symbolized in the form of a spin-flip process (valence-multiplet Auger). 


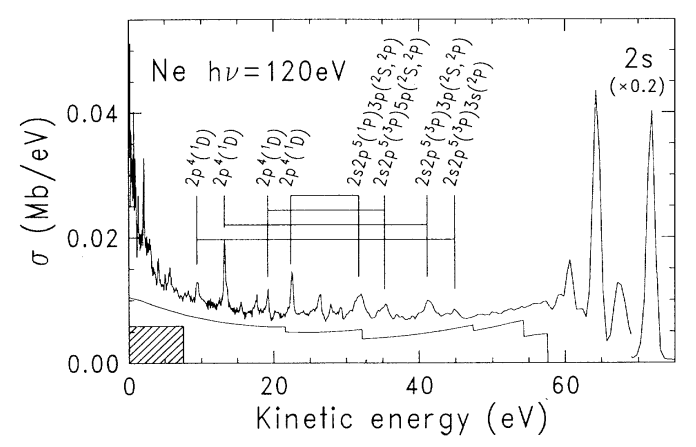

FIG. 2. Complete valence-electron spectrum of neon after photoionization with photons of $h v=120 \mathrm{eV}$. The most prominent satellite lines are depicted along with their corresponding Auger lines. The solid curves represent the distribution of shakeoff electrons stemming from simultaneous double ionization. The hatched area marks the energy region where valencemultiplet Auger decay occurs.

time-of-flight (TOF) detection of the emitted electrons was employed in order to guarantee high transmission and low background for low kinetic energy electrons. The duty cycle of 960 nsec of the single-bunch operational mode of DORIS II was particularly well suited to the flight lengths of $679 \mathrm{~mm}$ of our TOF spectrometer, because it makes it possible to record complete electron spectra down to near $0-\mathrm{V}$ kinetic energy. Details of the experimental setup are described elsewhere [9]. Figure 3 shows a complete electron spectrum taken at $h v=67 \mathrm{eV}$, an energy too low to allow excitation of inner-valence satellite states. Therefore the whole spectrum has to be the result of valence multiplet-changing Auger transitions. In this case the decay process has to involve a spin-flip or a more general multiplet-changing process, otherwise the transition would not be energetically allowed. In contrast to the high resolution shown by the Auger spectrum, the photosatellite part of the complete electron

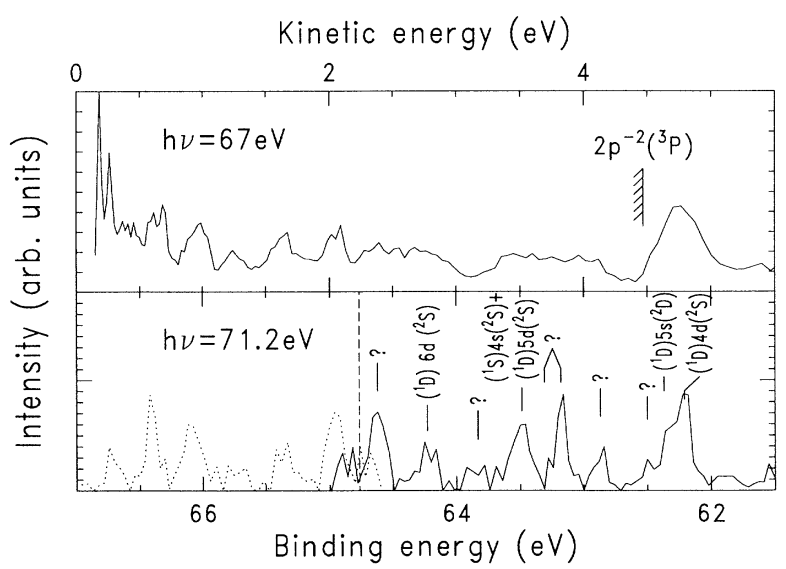

FIG. 3. Neon (a) valence electron spectrum taken at $h v=67$ $\mathrm{eV}$ and (b) high-resolution photoelectron spectrum from Ref. [10]. The dotted line represents the same spectrum but energetically inverted with respect to the lowest double-ionization threshold. spectrum is only poorly resolved because of the limited photon resolution achieved in this experiment in order to compromise between resolution and photon flux. As a result of this, all primary satellite lines are actually unresolved, inhibiting their identification.

However, the satellite part of this spectrum has been measured with high resolution very recently by Krause et al. [10]. The spectrum measured by these authors is shown in the bottom part of Fig. 3 along with the identifications given in Refs. [10,11]. The dotted line in this part of Fig. 3 represents the same spectrum but as a mirror image reflected at the center line between the first double-ionization threshold and $0-\mathrm{V}$ kinetic energy of the emitted electrons. Any real spectrum resulting from Auger decay of the observed satellite lines to this threshold should mimic more or less this hypothetical spectrum if one takes into account the continuous shakeoff electron distribution underneath the line structure [12]. The comparison between our measured Auger spectrum and the hypothetical Auger spectrum derived from the inverted high-resolution satellite spectrum shows indeed a great deal of similarity. In fact, after subtraction of the continuous shakeoff background each line of the Auger spectrum may be associated with an inverted photoelectron satellite line. In this way the identification of the different Auger lines seemed to be possible. However, in doing so, one is confronted with the fact that the strongest lines appearing in the spectrum are designated by Krause et al. [10] and Svensson et al. [11] to be $\left({ }^{1} D\right) n d\left({ }^{2} S\right)$ lines. The final ionic states of these lines are forbidden to decay to the $2 p^{-2}\left({ }^{3} P\right)$ double-ionization threshold for parity reasons. This is because within $L S$ coupling an even parity $2 p^{-2}\left({ }^{3} P\right) \epsilon l_{A}\left({ }^{2} S\right)$ final Auger state cannot be constructed. Consequently, no Auger decay should occur. Armen and Larkins [7] tried to escape the problem of these metastable $\left({ }^{1} D\right) n d\left({ }^{2} S\right)$ states by arguing that these states may possibly decay via a fluorescence Auger cascade. In such a process the first step would be a fluoresence decay to the $\left({ }^{1} D\right) m p\left({ }^{2} P^{o}\right)$ states, which could then further decay nonradiatively to the $2 p^{-2}\left({ }^{3} P\right)$ and $2 p^{-2}\left({ }^{1} D\right)$ final states. However, such a cascade process would result in Auger electrons having approximately $0.4 \mathrm{eV}$ less kinetic energy than they would have if the primary satellite state would decay directly. Considering the relative intensities as uneffected by this process, such an energy shift is not observed in our spectrum, although the resolution should be in principle good enough. In order to explain the surprisingly good agreement between satellite and Auger spectra we tried to look for an alternative interpretation. If we regard the designation of these satellite states, which have one decay branch only, as basically correct, and calculations done by Svensson [11] support this assumption, configuration interaction may be the only reason to allow the decay of these states. In order to prove which configuration could be the suitable candidates for such a mixing we performed calculations of relative energies and intensities of all possible Auger transition series using many-body perturbation theory (MBPT) as being shown schematically in Fig. 4. We found good agreement with the Hartree-Fock results of Armen and Larkins [8] for all cases they did 


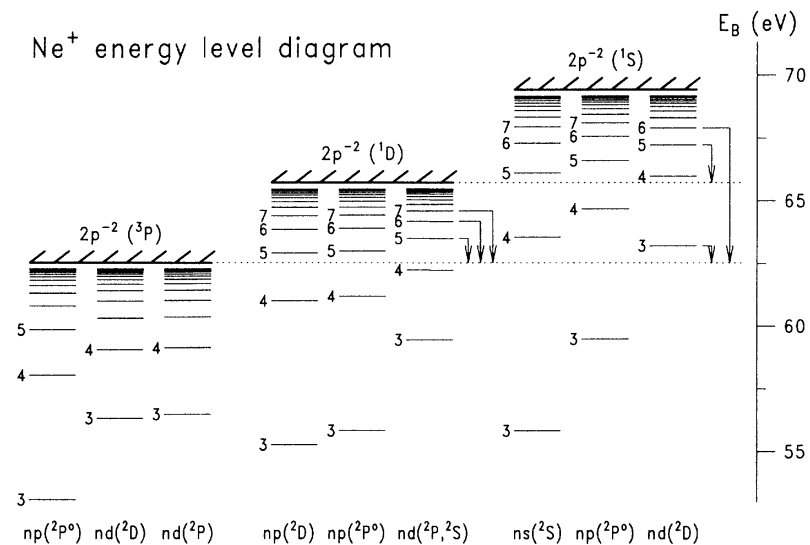

FIG. 4. Schematic energy-level diagram illustrating the possible valence-multiplet Auger decay modes in Ne. The energies of the different excitation series and their thresholds are derived from experimental data in all cases where such data do exist. The missing energy levels represent calculated values adjusted to the experimental data by quantum-defect theory. Some allowed transitions are shown explicitly; their corresponding Auger energy is indicated by the arrows.

calculate.

To make, for designation purposes, the results of our calculations more comparable with the experimental spectra we corrected the final- and initial-state energies with respect to the experimentally determined doubleionization thresholds and satellite line positions as far as they were known. Unknown positions were extrapolated using the quantum-defect formalism. For all multiplets of a certain configuration, particularly for those where no experimental data existed, we used the same average energy shift for their correction. The result is shown in Fig. 5 along with the background-subtracted Auger and in-

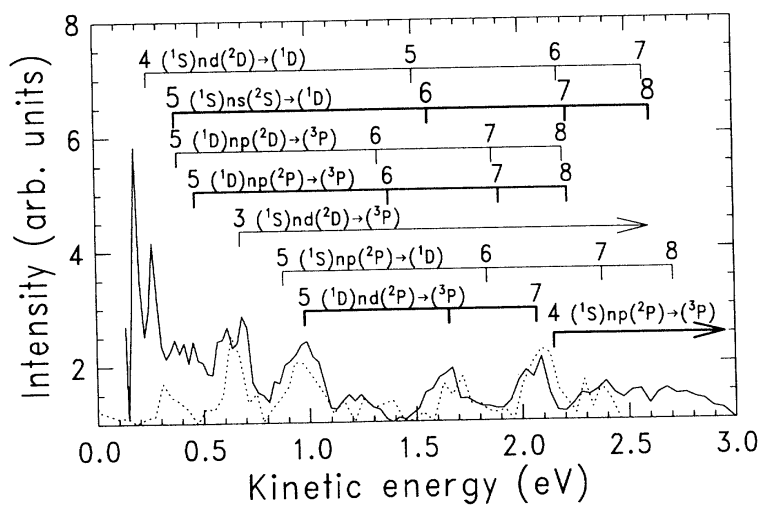

FIG. 5. Background subtracted Auger spectrum between 0 and $3 \mathrm{eV}$ from Fig. 3 along with the inverted high-resolution satellite spectrum of Ref. [10]. The designations derived according to Fig. 4 are shown as bars on top of the spectrum. The transition arrays with the highest initial-state population and decay rate are depicted by thicker bars.

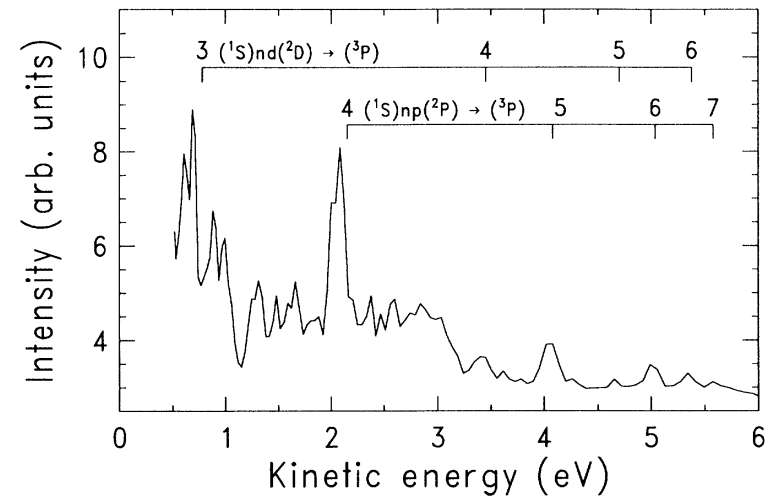

FIG. 6. The Auger part of a complete valence electron spectrum taken at $h v=73 \mathrm{eV}$ up to $6 \mathrm{eV}$ kinetic energy together with suggested designations.

verted satellite spectrum. The three intense Auger lines at $0.95,1.65$, and $2.05 \mathrm{eV}$ show best correspondence concerning their energy position with the transition $\left({ }^{1} D\right) n d\left({ }^{2} P\right) \rightarrow\left({ }^{3} P\right)$, as seen in Fig. 5. Assuming that the primary satellite states are basically $\left({ }^{1} D\right) n d\left({ }^{2} S\right)$ states that are stable against Auger decay, mixing with the $\left({ }^{1} D\right) n d\left({ }^{2} P\right)$ states may be the most likely mechanism for the observed transition pattern. The energy levels of both $L S$ series are very close to each other, promoting the changes for interaction between them. On the other hand, already a small degree of mixing between these states would finally result in the complete decay of the initially populated satellite states in the observed way, because the competing radiative processes are several orders of magnitudes less probable. In this sense our results show the existence of $L S$ mixing in the satellite states, requiring intermediate coupling for their correct description.

Most of the other structures in the spectrum could also be assigned to certain transitions, although many of them are too closely spaced to be resolved. However, the intensity distribution among the primary satellite states may help to specify the $\left({ }^{1} S\right) n s\left({ }^{2} S\right) \rightarrow\left({ }^{1} D\right)$, $\left({ }^{1} D\right) n p\left({ }^{2} P\right) \rightarrow\left({ }^{3} P\right), \quad$ and the above discussed $\left({ }^{1} D\right)$ nd $\left({ }^{2} P\right) \rightarrow\left({ }^{3} P\right)$ transitions as the most prominent ones.

Following the same scheme we were also able to designate the transitions of higher-lying Auger lines, particularly the ones between 2 and $6 \mathrm{eV}$. We assign these lines also as multiplet-changing transitions, in contrast to the lines with even higher kinetic energy, which are of the valence Auger type. The main transition arrays are $\left({ }^{1} S\right) n d\left({ }^{2} D\right) \rightarrow{ }^{3} P$ and $\left({ }^{1} S\right) n p\left({ }^{2} P\right) \rightarrow{ }^{3} P$, whose higher members may cause the small lines observed above $3 \mathrm{eV}$, as shown in Fig. 6.

In conclusion, we have studied the multiplet-changing valence Auger transitions in neon, both experimentally and theoretically. We show that the decay of the strongest satellite states being stable against Auger decay becomes possible by the mixing of different $L S$ states. Using calculated transition energies and decay rates we were able to assign most of the resolved structure in the experimental valence-multiplet Auger spectrum. 


\section{RAPID COMMUNICATIONS}

The authors are grateful to Professor F. P. Larkins and Dr. G. B. Armen for many helpful discussions, and to Dr. S. B. Whitfield for a critical reading of the manuscript. This work was supported by the Bundesminister für Forschung und Technologie under Con- tract No. 05414 CAB 7 and by the Deutsche Forschungsgemeinschaft under Contract No. Be 860/2-4. One of us (M.Y.A.) is indebted to the Humboldt foundation for financial support.
*Permanent address: Tomsk Polytechnic University, Tomsk, 634004, Russia.

[1] P. Lablanquie, in Proceedings of the Seventeenth International Conference on the Physics of Electronic and Atomic Collisions, Brisbane, Australia, edited by W. R. MacGillivray, I. E. McCarthy, and M. C. Standage (Hilger, Bristol, 1992), p. 507.

[2] U. Becker and R. Wehlitz, Phys. Scr. T41, 127 (1992).

[3] G. King, Phys. Scr. (to be published).

[4] U. Becker, R. Wehlitz, O. Hemmers, B. Langer, and A. Menzel, Phys. Rev. Lett. 63, 1054 (1989).

[5] S. D. Price and J. H. D. Eland, J. Phys. B 22, L153 (1989).

[6] A. A. Wills, A. A. Cafolla, A. Svensson, and J. Comer, J.
Phys. B 23, 2013 (1990).

[7] G. B. Armen and F. P. Larkins, J. Phys. B 24, 741 (1991).

[8] G. B. Armen and F. P. Larkins, J. Phys. B 25, 931 (1992).

[9] U. Becker, D. Szostak, H. G. Kerkhoff, M. Kupsch, B. Langer, R. Wehlitz, A. Yagishita, and T. Hayaishi, Phys. Rev. A 39, 3902 (1989).

[10] M. O. Krause, S. B. Whitfield, C. D. Caldwell, and J.-Z. Wu, J. Electron Spectrosc. Relat. Phenom. 58, 79 (1992).

[11] S. Svensson, B. Eriksson, N. Mårtensson, G. Wendin, and U. Gelius, J. Electron Spectrosc. Relat. Phenom. 47, 327 (1988).

[12] R. Wehlitz, F. Heiser, O. Hemmers, B. Langer, A. Menzel, and U. Becker, Phys. Rev. Lett. 67, 3764 (1991). 